To list an event in the Calendar, contact J. Dininny,

Materials Research Soclety, 9800 McKnight Road.

Pittsburgh, PA 15237; (412)

367-3036; tax (412) 367-4373.

MIRIS identffles meetings sponsored or co-sponsored by the Materlals Research Society; boldface type without the logo Identlities MRS-endorsed meetings.

See MRS BULLETIN Vol. XV No. 5 for Calendar events from June 1 through June 30, 1990.

\section{JULY 1990}

2.5

7th CIMTEC Satellite Symposium

4. Hight- $\mathrm{T}_{\mathrm{s}}$ Superconductors

(See June 24-30 CIMTEC listing)

Trieste, lialy

7th CIMTEC, Satellite Symposium

4, P.O. Box 174, 48018

Faenza, Italy: 546-664143;

$\operatorname{tax} 546-664138$

8-13

Intemational Symposium on

Optical and Optoelectronic

Applied Science and Engineening

San Diego, CA

SPIE, P.O. Box 1Q, Bellingham,

WA 98227-0010; (206) 676-3290;

$\operatorname{tax}(206)$ 647-1445

$9-12$

Conference on Nondestructive

Evaluation of Modem Ceramics

American Society for Nondestruc-

tive Testing, 4153 Arlingate Plaza,

P. O. Box 28518. Columbus, $\mathrm{OH}$

43228; tax (614) 274-6899

$9-13$

6th International Conference on

Valence Fluctuations Ni-ICVF

Rio de Janerio, Brazil

G.E. Garberis, Instituto de Fisica

"Gleb Wataghin," UNICAMP

Cidade Universitaria, Barao

Geraldo, Caixa Postal 6165, 13081

Campinas (S.P.). Brazil: PABX

(0192) 39.1301

10-12

Nodestructive Evaluation of

Modem Ceramics

Columbus, $\mathrm{OH}$

B. Runyon, American Society for

Nondestructive Testing, 4153

Aritingate Plaza, Columbus, $\mathrm{OH}$

43228-0518; (800) 222-2768

11-13

Optoelectronics Conference

Chiba, Japan

K. 1to, OEC ' 90 , do Business

Center for Academic Societies

Japan, Conference Dept.,

Crocevia Hongo 2F, 3-23-1

Hongo, Bunkyo-ku, Tokyo 113,

Japan: 81-3-817.5831; tax 81.3.

817.5836
$15-20$

Review of Progress in

Quantitative NDE

a Jolla $\mathrm{CA}$

D.0. Thompson, lowa State

University, Center for NDE

Applied Sciences Center II, 1915

Scholl Rd. Ames, IA 50011; (515)

2947803; $\operatorname{tax}$ (515) 294.777

$15-20$

8th American Conference on

Crystal Growth

Viil $\mathrm{CO}$

B.L. Sopori, Solar Energy

Research Institute, 1617 Cole

Blvd. Golden, CO 804073393.

(303) $231-1383$

$16-20$

Conference on Nonlinear Optics:

Materials, Phenomena, and

Devices

Kauai, Hawaii

G. MCBride, IEEE, 345 E. 47th

St., New York, NY 10017-2394;

(201) 562.3896

17.19

Low Temperature Engineering and

Cryogenics Conference, LTEC'90

Southampton, England

Cryogenics Conterence Office,

Institute of Cnogenics, University

of Southamoton, SOS SNU

United Kingdom; 0703-595000,

ext. 2059 or 2662 ; tax 0703

593939

22-27

13th Intemational Liquid Crysta

Conference

Vancouver, Canada

B. Bergersen, Dept. of Physics,

Univ. of British Columbia.

Vancouver, V6T 246 Canada;

(604) 228-2603

22.3

NATO Advanced Stuoy Institute on

Diamond and Diamond-Like Films

Castelvecchio Pascoli, Italy

C.J. McHargue Metals and

Ceramics Div., Oak Pidge National

Lab., P.0. Box 2008, Oak Ridge.

TN 37831-6118; (615) 574-4344;

$\operatorname{tax}(615)$ 5747659

23-26

Conference on Advances in

Cementitious Materials

Gaithersburg, MD

Amenican Ceramic Society, 757

Broksedge Plaza Orive Wester

ville, OH 43081; (614) $890-4700$

$\operatorname{tax}(614) 899-6109$

23-27

5 th Intemational Conference on

Scanning Tunneling Microscopy/

Spectroscopy and 1st intema-

tional Conference on Nanometer

Scale Science \& Technology

Baltimore, MD

IS. Murday, Code 6100 Naval

Research Lab., Washington, DC

20375-5000; (202) 767-3026; tax

(202) 4047139
24-26

Conference on Polymer Biends

Cambridge, United Kingdom

OC. Varley, Conf. Mgr. PRI, 11

Hobart Place, London

SWIW OHL

263

8th Intemational Conference on

Ion Implantation Technology

University of Surrey, United

Kingdom

M. Jackson, Dept. of Electronic and Electrical Engineering, Univ. of Surrey, Guildtord, Surrey GU2 5XH, United Kingdom; 44-483.

509133; tax 44-483-34130

29-3

37th International Field Emission

Symposium

Albuquerque, NM

G.L. Kellogg, Div. 1114, Sandia

National Laboratories, Albuquer que, NM 87185

$30-1$

Research Trends in Superconductivity

La Jolla, CA

Inst. for Adv. Phys. Studies, La Jolla intt. School of Physics, P. Box 2946, La Jolla, CA 92038 ; (619) $456-5737$

30-1

Powder Metallurgy: Key to

Advanced Materials Technology

Vancouver Canada

Member/Customer Service

Center ASM Intemational, Metals

Park OH 44073; (216) 3325151: $\operatorname{tax}$ (216) $338-4634$

$30-2$

8th International Conference

on lon Implantation Technology

University of Sumey, United

Kingdom

P.L.P. Hemment, Dept of

Electronic \& Electrical Engineer-

ing, Univ. of Surrey, Guilftond

Surrey GU2 5XH; (0483) 571281:

$\operatorname{tax}(0483) 300803$

$30-3$

POLYSE'9O: Polycrystalline

Semiconductors

Schwäbisch Hall, W. Gemany

H.P. Strunk, Max-Planck Institute

FKF, Heisenbergstr. 1, 07000

Stuttgart 80, FRG; (711) 6860.

645; $\operatorname{tax}$ (711) 6874371; or J.H.

Wemer Max-Planck-Institute.

Heisenbergstr. 1, 7000 Stuttgart

80. W. Germany; (49) 711/6860

645

$30-3$

5th Intemational Conference on

the Physics of Electro-Optic

Microstructures and Microdevices

Heraklion Crete, Greece

A. Christou. Code 6830 Naval

Research Laboratory 4555

Overtook Ave. SW, Washington.

DC 20375-5000; tax (202)

767-0546
303

Particulate Ceramics Processing:

The Future for Manutacturing

Cambridge, MA

Director of the Summer Session,

M.I.T. Rm. E19356, Cambridge,

MA 02139; (617) 253-2101

$31-2$

4th intemational Conterence on

Shallow Impurities in Semicon

ductors

London, United Kingdom

Davies, Physics Department,

Kings College London, The

Strand, London WC2R aLS

United Kingdom

\section{AUGUST 1990}

6-10

20th International Conference on

the Physics of Semiconductors

Thessabriki, Greece

NA. Economou, P. O. Box 19615

54006 Thessabnili, Greece; (30)

31-991439; $\operatorname{tax}(30)$ 31-214276

8-10

Specialty Polymers 'SO

Baltimore, MO

W. Harvey, SP '9Q, Butterwortt

Scientific Ltd., P.O. Box 53,

Westbury House, Bury St.,

Guilftord Surrey GU2 58H

United Kingdom; 44-483-300966;

fax 44-483-301563

$12-18$

12th Intemational Congress for

Electron Microscopy

Seattle, WA

R.M. Fisher, Univ. of Washington,

5001 25th Ave. NE, GH-22,

Seattle. WA 98195; tax (206)

545.9359

13-15

LF19 Satelitite Conference on High

Temperature Superconductivity

Cambridge, United Kingdom

K.S. Ditfey, IRC in Super-

conductivity, Madingley Rd

Cambridge C83 OHE, UK: (223)

337472; $\operatorname{tax}(223)$ 337074

13-17

7th Intemational Conference on

Rapidly Quenched Materiats

Stockholm, Sweden

Dept. of Casting of Metals, Royal

Institute of Technology, S-100 44

Stockholm, Sweden; $468-79079$

84: tax 46-8109199

$16-21$

Intemational Conference-

Chemistry of Electronic Ceramic

Materials

Targhee National Forest, Wr

RS. Roth, NIST, Ceramics Div.

420 Bdg. 223, Rm. 2216.

Gaithersburg, MD 20899; (301)

975-61t6; $\operatorname{tax}(301)$ 975-2128

$20-24$

3rd Electronic Materials \&

Processing Congress

San Francisco, CA

ASM Intemational, Materials Park

OH 44073; (216) 338-5151; tax

(216) $338-4634$

21-23

Biointeractions ' $90-\mathrm{New}$

Biomaterials for the Nineties 
$4-8$

EPS-8, Trends in Physics Amsterdam, The Netherlands Conference Secretariat, c/o RA Organisatie Bureau Amsterdam bv, Europaplein 12, 1078 GZ Amsterdam, The Nethertands; 31 20-5491212; $\operatorname{tax} 31-20-464469$ 5-7

Analysis by a Combination of lon Beam (Accelerator-Based) and Surface Specific Techniques Namur, Belgium

G. Demortier, Département de

Physique. Facultes Universitaires N-D de la Paix, ne de Bruxelles, 61, B.-5000 Namur, Belgium; fax 32-81-230391

6-22

14th International School of Theoretical Physics: Strongly Comelated Electron Systems and Hight $T_{c}$ Superconductivity Szczyrk, Poland

E. Zipper, Dept. of Theoretical

Physics, University of Silesia Uniwersytecka 4, PL-90 007: Katowice, Poland; 48-32-588431 or 588211 ext. 409 ; tax $48-32$. 599605

$9-14$

MIRIS Ion Beam Modification of Materials-IBMM ' 90

Knoxville, TN

P.H. Green, IBMM '9a, Oak Ridge

National Lab., Solid State Div.

P.0. Box 2008, M.S.6033, Oak

Ridge, TN 37831-6033;

(615) 576-1864; $\operatorname{tax}(615)$

$574-4143$

(See related article in

Vol. XVNo. 12.)

10-13

1st Intermational Conference on

Micro System Technologies

Berlin, Germany

Technische Universität Berlin, FSP

Technologien der Mikroperipherik,

Sekr EN 4, Einsteinufer 17, D-

1000 Berlin 10 . W. Germany;

(030) 314-2 35 53; $\operatorname{tax}(030)$

$313-6733$

10-13

2nd World Congress on

Superconductivity

Houston, TX

Worid Congress on Super-

conductivity P. O. Bax 27805

Houston, TX $77227-7805 ;$; 713 )

895-2500: $6 \times(713) 623-3560$

10-14

2nd Intemational Conference on

Plasma Surtace Engineering

Garmisch-Partenkirchen, W.

Germany Deutsche Gesellschaft

fir Gavano-u Oberflächentech-

nik e. V. Horionplatz 6, D-4000

Dusseldorf $1, W$. Germany:

49-211-132381

10-20

NATO Advanced Study Institute on Applications of Metallic and Ceramic Superconductivity

Fort Collins, $\mathrm{CO}$

H. Weinstock, AFOSR/NE, Bolling AFB, Washington, DC 20332

6448; (202) 767-4933; tax (202)

$767-0466$
11-12

Workshop on High-Density

Plasma Techniques and Proc-

esses for Integrated Circuit

Fabrication

Burlingame, $C A$

Continuing Education in Engineer-

ing, UC Berkeley Extension, 2223

Fulton St., Berkeley, CA 94720;

(415) 642-4151; tax (415)

$643-8683$

11-13

3rd Intemational SAMPE Metals

Conference

Wiliamsburg, VA

N. Hawley, 1055 W. San Bemar-

dino Rd., Covina, CA 91722;

(818) 331-0616

17-19

\section{MIRIS 2nd Intemational}

Conference on Electronic

Materials (ICEM-II)

Hoboken, NJ

J. Stokes, Materials Research

Society, 9800 McKnight Rd.

Pittsburgh, PA 15237; (412) 367-

3003; $\operatorname{tax}(412) 367-4373$

(See related article in

VbI. XNNo. 12.)

$17-20$

Applied Optics and Opto-

Electronics

University of Nottingham, United

Kingdom

Applied Optics Group, Blackett

Laboratory, Imperial College,

London SW7 2BZ; (01) 5895111

ext $6975 / 6842$

18-20

Microcircuit Engineering ' 90

Letsen, Belgium

K. Legein, IMEC VZw, Kapeldreef

75, 8-3030, Leuven, Belgium

18-20

4th Annual Conference on

Superconductivity and

Applications

Buffalo, NY

Y.H. Kao, NY State Institute on

Superconductivity, 330 Bonner

Hall, State Univ. of New York at

Buffalo, Buffalo, NY 14260; (716)

636-3463; $\operatorname{tax}(716) 636-2528$

23-27

MRS 2nd Intemational

Conterence on the New

Diamond Science and

Technology

Washington, DC

ICNDST, cJo LRW Associates,

1218 Baffour Drive, Amold, MD

21012; (301) 647-5136

(See related article in

Vol. XV, No. 3.)
23-27

30th National Congress of SECV Santiago de Compostela, Spain xx Congreso Nacional de Sociedad Espanola de Cerámica y Vidrio, Carretera de Valencia, $\mathrm{Km}$. 24,300,28500 Arganda del Rey Madrid, Spain; (91) $8711800-04$

24-27

European Gallium Arsenide

Conference

St. Helier, Jersey, Channel islands

Meetings Officer, Institute of

Physics, 47 Belgrade Square

London SWIX 80X, England

$24-28$

1990 Applied Superconductivity

Conterence

Aspen, CO

ASC190, Centennial Conferences,

5353 Manhattan Circle, Suite 103

Boulder CO 80303; (303)

$499-2299$

25-26

Use of Composite Materials in

Load Bearing Marine Structures

Artington, VA

A. Stavowy National Research

Council, Marine Board HA250,

2101 Constitution Ave. NW,

Washington, DC 20418; (202)

3343119; $\operatorname{tax}(202)$ 334-2620

30-5

Microelectronic Processing

Integration

Santa Clara, CA

SPIE, PO Box 10, Bellingham

WA 98227-0010; (206) 676-3290;

$\operatorname{tax}(206)$ 647-1445

OCTOBER 1990

1-4

11th European Conference on

Surface Science (ECOSS-11)

Salamanca, Spain

ECOSS-11, Laboratorio Fisica de

Superficies, Instituto Ciencia de

Materiales C.S.I.C., Serrano 144,

E-28006 Madnid, Spain; $34-1$

2618805 ext. 224: tax 34-1-

4117651

2.3

4th Annual Alabama Materials

Research Conterence

Tuscaloosa, AL

D.M. Stefanescu, University of

Alabama, Metalfurgical \&

Materials Engineering, Box

870202. Tuscaloosa, AL 35487-

0202; (205) 348-1749; $\operatorname{tax}(205)$ $348-8573$

3-6

American Society of Composites: Fourth Technical Conference on Composite Materials

Blacksburg, VA

M.W. Hyer, RSM Dept., Virginia

Tech., Blacksburg, VA 24061;

(703) 231-5372
7-11

TMS Fall Meeting: Physical Metallurgy and Materials

Detroit, $M$

TMS, 420 Commonwealth Dr.

Warrendale, PA 15086; (412) 776 -

9050; $\operatorname{tax}(412) 776-3770$

8-12

37th National Vacuum

Symposium

Toronto, Ontario, Canada

M. Churchill, American Vacuum

Society, 335 E 45th St. New

York, NY 10017; (212) 661-9404

14-19

178th Meeting of The Electro-

chemical Society

Seattle, WA

The Electrochemical Society 10

S. Main St., Pennington, NJ

08534-2896; (609) 737-1902

15-17

ISHM '90: International Sympo-

sium on Microelectronics

Chicago, IL

N. Binkley, ISHM, P.O. Box 2698 ,

Reston, VA 22000-2698; (703)

471-0066; $\operatorname{tax}(703)$ 471-1937

21-24

11th Intemational Workshop on

Rare-Earth Magnets and Their

Applications

Pittsburgh, PA

S.G. Sankar, Camegie Mellon

University, Mellon Institute, 4400

Fifth Ave., Pittsburgh, PA 15213:

(412) 268-5649; $\operatorname{tax}$ (412) 268 3101

$22-24$

Wrkshop on Tungsten and Other

Advanced Metals for ULS

Applications VII

Dallas, $\mathrm{TX}$

Continuing Ed. in Engineering,

Univ. of California Extension,

2223 Fulton St., Berkley, CA

94720; (415) 642-4151; fax (415) $643-8683$

6th Intemational Symposium on

Magnetic Anisotropy and

Coencivity in Rare-Earth-Transition

Metal Alloys

Pittsburgh, PA

S.G. Sankar, Camegie Mellon

University Mellon Institute, 4400

Fifth Ave., Pittsburgh, PA 15213;

(412) 268-5649; $\operatorname{tax}(412)$

$268-3101$

25-27

Technology for Tomorrow

Seattle WA

S. Laurich-McIntyre, Pacific

Norttwest Section, American

Ceramic Society, clo University of

Washington, Roberts Hall FB-10

Seattle, WA 98115; (206)

$781-2663$

$27-2$

Intemational Conference on

Physical Concepts of Materials for

Novel Optoelectronic Device

Applications

Aachen, W. Germany

SPIE, P.O. Box 10, Bellingham,

WA 98227-0010; (206) 676-3290 $\operatorname{tax}(206)$ 647-1445

$28-31$ 
13-15

2nd International Ceramic

Science and Technology Congress Orlando, $\mathrm{FL}$

American Ceramic Society, 757

Brooksedge Plaza Drive, Westerville, $\mathrm{OH} 43081$; (614) 890-4700; $\operatorname{tax}(614) 899-6109$

13-15

ACerS Electronics Division

Meeting

Ortando, FL

American Ceramic Society, 757

Brooksedge Plaza Drive, Wester-

ville, $\mathrm{OH} 43081$; (614) 890-4700

$\operatorname{tax}(614) 899-6109$

13-15

5 th Annual National Educators'

Workshop: Update 90

Gaithersburg, MD

J.A. Jacobs, School of Technot

ogy, Norfolk State Univ., 240

Corprew Ave., Nortolk, VA 23504:

(804) $683-8109 / 8712$

(See related article in

bot. N No. 4)

$26-1$

MIRIS Materials Research

Soclety Fall Meeting

Boston, MA

M. Geil, Materials Research

Sociely, 9800 McKnight Rd.

Pittsburgh, PA 15237; (412) 367 -

3003: $\operatorname{tax}(412) 367-4373$

26-7

Experimental Workshop on High-

Temperature Superconductors

and Related Materials (Advanced

Activities)

Trieste, Italy

International Center for Theoreti-

cal Physics, P.0. Box 586, 1 -

34100, Trieste, Italy; 22401; fax

224163

27-30

E-MRS European Materials

Research Soclety Meeting

Strasbourg, France

P. Siffert, Centre de Recherches

Nucleaires, Laboratoire PHASE,

67037 Strasbourg. Cedex

France; (88) 286543 ; tax (88)

280990

\section{JANUARY 1991}

13-18

Mechanical Fatigue in Advanced

Materials

Santa Barbara, CA

R.J. Yacyshyn, Engineering

Foundation, 345 E. 47th St. New

York, NY 10017; (212) 705-7835

$29-1$

PCSI 18: Physics and Chemistry

of Semiconductor Interfaces

Long Beach, CA

R.W. Grant, Rockwell Interna-

tional Science Center, P.O. Box

1085, Thousand Oaks, CA 91358;

(805) 373-4219

\section{FEB}

10-15
POLYMER 91-POlymer Materi-

als: Preparation, Characterization

and Properties

Melboume, Australia

G.B. Guises, RACI Polymer Div.

P.0. Box 224, Belmont. Victoria

3216 Australia; 052472695 ; fax 052472657

$16-22$

OFC-Optical Fibers Communications Conference

San Diego, CA

Lasers and Electro-Optics Society,

445 Hoes Lane, P.O. Box 1331.

Piscataway NJ 08855-1331; (201)

562-3895; tax (201) 562-1571

17.20

14th Annual Meeting of the

Adhesion Society

Cleanwater, FL

H.M. Clearfield, IBM T.J. Watson

Research Center, P.0. Box 218 ,

MS 38-145, Vorktown Heights

NY 10598; (914) 945-3202

17-21

TMS Annual Meeting

New Orteans, LA

TMS, 420 Commonwealth Dr.

Warrendale, PA 15086; (412) 776 -

9050: $\operatorname{tax}(412) 776-3770$

28-1

Washington Materiats Forum:

Superconductors and Semicon-

\section{ductors}

Washington, DC

Materials Research Society, 9800

Mcknight Rd., Pittsburgh, PA

15237; (412) 367-3000; fax (412)

$367-4373$

\section{MARCH 1991}

$12-13$

Bonding and Repair of

Composites II

Zurich, Switzerland

K. Royle, Rapra Technology Ltd.,

Shawbury, Shrewsbury, Shrop-

shire SY4 4NR, England; 44939-

250383; tax 44-939-251118

$17-2$

7th International Symposium on

Halide Glasses

Lome, Victoria, Australia

D.R. MacFartane, Centre for

Advanced Materials Technology,

Monash University, Clayton,

Victoria 3168, Australia; fax 61-3. 5654998

18-22

American Physical Society

Meeting

Cincinnati, $\mathrm{OH}$

APS, 335 E. 45th St., New York.

NY 10017; (212) 682-7341

24-27

3rd European Workshop on

Refractory Metals and Silicides

Stockholm, Sweden

C.S. Petersson, KTH, Box 1298 ,

S-164 28 Kista-Stockholm

Sweden; (46) 8-752 1401; $\operatorname{tax}(46)$

8-752 7782
25-28

7th Oxtord Conference on

Microscopy of Semiconducting

Materials

Oxtord, United Kingdom

A.G. Cullis, Royal Signals \& Rada

Establishment, St. Andrews Rd.

Malvem, Worcs WR14 3PS,

United Kingdom

APRIL 1991

7-11

Intemational Conterence on

Wear of Materials-91

Ortando, $\mathrm{FL}$

L. Friedman, Meetings Dept.

ASME, 345E. 47th St., New

York, NY 10017-2304

(212) $705-7722$

(See related articte in

VI. XN No. 12.)

15-17

Frontiers of Tribology

Strattord Upon Avon, United

Kingdom

Meetings Office, Institute of

Physics, 47 Belgrave Square,

London SW1X 80X:

44-1-235-6111

$16-18$

Magneto-0ptical Recording

Intemational Symposium ' 91

,Tokyo, Japan

T. Suzulo, IBM Almaden Research

Center, 650 Hary Rd., San Jose,

CA 95120-6099; (408) 927-2099;

tax (408) 927-2100

21-24

Euro MBE-91: 6th European

Conference on Molecular Beam

Epitaxy and Related Growth

Methods

Tampere, Finland

R. Siekkinen, Euro MBE-91,

Tampere University of Technology,

P.O. Box 527, SF-33101, Tampere,

Finland; 358-31-162441; fax 358-

31-162164

$29-3$

MIRIS Materials Research

Society Spring Meeting

Anaheim, CA

M. Geil, Materials Research

Society, $\mathbf{9 8 0 0}$ McKnight Rd.

Pittsburgh, PA 15237; (412) 367-

3003: $\operatorname{tax}(412) 367-4373$

MAY 1991

5-11

3rd European Conference on

Crystal Growth

Budapest, Hungary

E. Lendvay, Research inst. for

Technical Physics, Hungarian

Acad. of Science, Ujpest 1, PF

76. Budapest. Hungary 1325:

(36) (1) 698-037

8-10

75th Arniversary Symoosium on

Metallography in conjunction with

ASTM Standards Meeting

Attantic City, NJ

ASTM, 1916 Race St. Philader

phia, PA 19103-1187; (215)

299-5400

13-17

CLEO '91: Conterence on Lasers and Electro-0ptics

Battimore, MD

Lasers and Electro-Optics Society

445 Hoes Lane PO Box 1331 .

Piscataway, NJ 08855-1331; (201)

562-3895; $\operatorname{tax}$ (201) 562-1571

\section{JUNE 1991}

17-20

6th Japan Institute of Metals

Symposium on Intermetallic

Compounds

Sendai, Japan

0 . Iumi, Institute for Material

Research, Tohoku Univ., Katahira,

Sendai 980 , Japan; 81-22-227-

6200; tax 81-22-264-7984

$18-20$

5th Intemational SAMPE

Electronic Materials and Proc-

esses Conference

Los Angeles, CA

SAMPE Business Office, P.O. Box

2459, Covina, CA 91722; (818)

331-0616; $\operatorname{tax}(818) 332-8929$

18-21

Electronic Materials Conference

Boulder, CO

TMS, 420 Commonwealth $0 \mathrm{r}$.

Warrendale, PA 15086; (412) 776

9050; $\operatorname{tax}(412) 776-3770$

1921

Conference on Fracture Proc-

esses in Brittle Disordered

Materials

Noordwijk, The Netherlands

Congress Office ASD, P.O. Box

54,2640 AB Piinacker, The

Nethertands; 31-17-365356; tax

31-17-362242

\section{JULY 1991}

21-26

American Crystallographic

Association Annual Meeting

Toledo, $\mathrm{OH}$

M.C. Etter, Dept. of Chemistry,

Univ. of Minnesota, 78 Kolthof

Hall, Minneapolis, MN 55455;

(612) 624-5217

\section{2-24}

Euromat 91: 2nd European

Conference on Advanced

Materials and Processes

University of Cambridge, United

Kingdom

Euromat 91, Conference Dept.,

The Institute of Metals, 1 Cartton

House Terrace, London. SWIY

5DB United Kingdom; 4471-

8394071; tax 44-71-8392289

\section{$22-26$}

Intemational Conference on

Materials and Mechanisms of

Supenconductivity - High

Temperature Superconductors

(MFS-HTSC III)

Kanazawa, Japan

K. Kitazawa, Dept. of Industria 